\title{
RFI Environment at the Shanghai Sheshan Site
}

\section{Zhi-Qiang Shen*, Bin Li and Tao An}

Shanghai Astronomical Observatory

E-mail: zshendshao.ac.cn, bingeshao.ac.cn, antaodshao.ac.cn

\begin{abstract}
At the time Shanghai Sheshan 25 meter radio telescope was built in 1987, the local radio environment was good. With the increasing interference from the active users of the radio spectrum, the issue of how to protect radio astronomical observations from RFI becomes very serious. This is getting even more demanding with the construction of a new 65 meter in diameter radio telescope at the same region, which will operate in a wide frequency range (from $1.4 \mathrm{GHz}$ to $43 \mathrm{GHz}$ ). As the first step, we have performed a RFI monitoring for the site evaluation since 2008. We will report on these assessments along with other related topics of the RFI protection at the Shanghai Sheshan telescope site.
\end{abstract}

RFI mitigation workshop

29-31 March 2010

Groningen, the Netherlands

\footnotetext{
* Speaker.
} 


\section{Introduction}

It has been almost 80 years since the Jansky's first detection of extraterrestrial radio emission at a frequency of $20.5 \mathrm{MHz}$ from the Milky Way in August 1931 with his rotatable directional antenna system (now known as "Jansky's merry-go-around"). Jansky's discovery laid the groundwork for the development of radio astronomy. As a result, the radio window is the first non-optical window in the electromagnetic spectrum and, has turned out to be a very useful band for not only the astronomical research but also for its application in many other fields, such as radio telecommunication.

In the early days, as the passive users of radio spectrum, radio astronomers enjoyed relatively easy and interference free access to (almost) the whole radio band. However, the situation has been gradually changed with the trend of the significant increase in the demand and availability of many commercial applications of radio spectrum. Such an influence of the active services (in spite of their origin) on radio astronomy is referred to as the radio frequency interference (RFI). This raises the issue of RFI mitigation, in general, to separate cosmic signals from man-made transmissions.

As the first step of our effort to deal with RFI, radio astronomers should have better knowledge of RFI environment around the site of their radio telescopes. This would allow us to better define scientific goals to make the maximum use of the very sensitive radio telescopes as the passive instruments. Mainly for this reason, we have carried out a survey of RFI at the location of the Shanghai 65 meter radio telescope, which is now under construction at Shanghai Sheshan territory. We will first present a brief introduction to the project of the Shanghai 65 meter radio telescope, and then discuss the RFI survey assessment along with some other related topics of the RFI excision at the Shanghai Sheshan telescope site.

\section{Shanghai 65 Meter Radio Telescope}

The Shanghai Astronomical Observatory was funded in 2008 by the Chinese Academy of Sciences and the Science and Technology Commission of Shanghai Municipality to build a 65 meter radio telescope for both space-related and radio astronomical observations. The project was supported by the Shanghai government to locate the telescope at Shanghai Sheshan area, in the same region the first Chinese VLBI station of a 25 meter in diameter radio telescope was constructed in 1987.

The Shanghai 65 meter radio telescope is designed to be a general purpose, fully steerable antenna. It will have a main reflector of 65 meter in diameter in a shaped Cassegrain configuration with a primary focal ratio of 0.32 . The primary active surface control system will be installed to compensate (mainly) the repeatable gravitational deformations to maintain the nominal shape of the primary reflector, and then to reach the best performance even at high frequencies. The main structural constraints for the antenna design are the surface accuracy and the pointing accuracy. The primary reflector consists of a total of 1008 aluminum panels in 14 rings, and at its back about 1112 electromechanical actuators will be mounted at each corner of four adjacent panels. A custom actuator design is now under way. The telescope is currently designed to operate to $15 \mathrm{GHz}$ without use of the active surface system. The antenna will be equipped with receivers to cover 8 frequency bands from $1.4 \mathrm{GHz}$ to $43 \mathrm{GHz}$, including L-, S-, C-, X-, Ku-, K-, Ka-, and Q-band. Among those, 


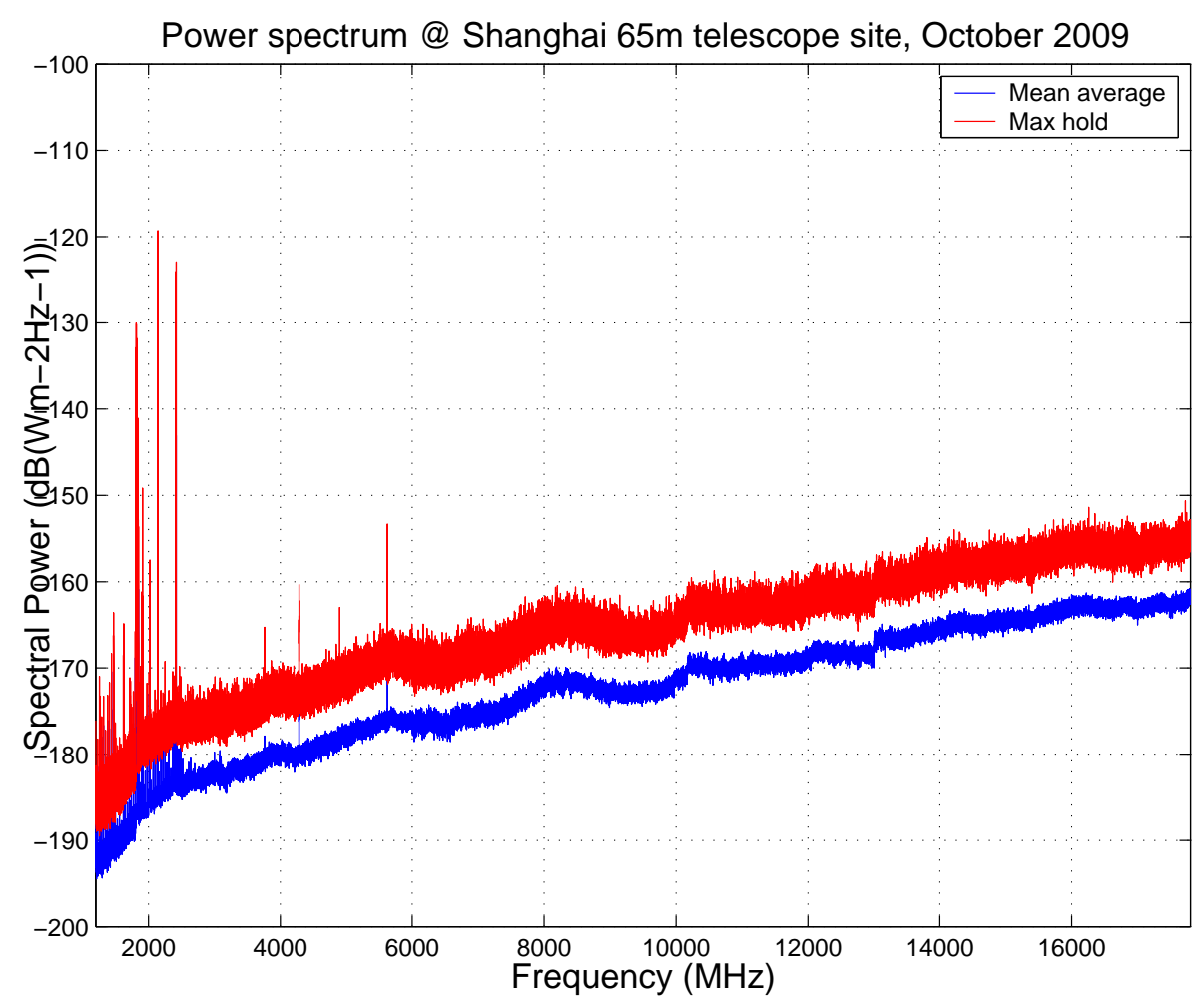

Figure 1: RFI spectrum for the Shanghai Sheshan telescope site

both $\mathrm{S} / \mathrm{X}$ and $\mathrm{X} / \mathrm{Ka}$ are dual-frequency bands. The Shanghai 65 meter radio telescope now is in its full construction phase and will be completed in 2012.

\section{RFI Environment at the Shanghai Sheshan Site}

At the time Shanghai Sheshan $25 \mathrm{~m}$ radio telescope was built in 1987, the local radio environment was good. The new 65 meter telescope will be located at about $6 \mathrm{~km}$ west to the 25 meter telescope. However, two decades have passed since the construction of the 25 meter telescope. To gain a better understanding of the current RFI environment at the Sheshan area, we started a RFI monitoring during the planning phase of the Shanghai 65 meter telescope. The first assessment was done by the Shanghai Radio Administrative Bureau in May 2008. The RFI survey covered a frequency range from $1 \mathrm{GHz}$ to $40 \mathrm{GHz}$. The results showed that there are some interference at the lower frequency L- and S-band, but most RFI appears at the bands outside of the designed frequency range for the 65 meter telescope and, at C-band and above RFI is not a problem. For example, our S-band range is $2.15-2.45 \mathrm{GHz}$ to avoid the $3 \mathrm{G}$ mobile band around $2.1 \mathrm{GHz}$. But, RFI at about $2.4 \mathrm{GHz}$ is likely to be related to various WiFi system. And a particular attention should be paid to a strong RFI at $1.47 \mathrm{GHz}$ which falls in the designed L-band of $1.25-1.80 \mathrm{GHz}$.

More recently, a team composed of engineers and astronomers from Beijing and Shanghai performed more thorough RFI measurements at Shanghai 65 meter telescope site. This time, we concentrated on transmissions band between $1 \mathrm{GHz}$ and $17.8 \mathrm{GHz}$. Both the vertical and horizontal 
polarizations from four directions (East, South, West, and North) were measured. The basic RFI monitoring system contains an antenna, low noise amplifiers (LNAs), filter, coaxial cable, and spectrum analyzer. A double-ridged waveguide horn antenna was used in the measurement, which has more than $10 \mathrm{dBi}$ uniform gain between $1 \mathrm{GHz}$ to $17.8 \mathrm{GHz}$ frequency range. Two LNAs were adopted to improve the detection sensitivity of the receiver system. One LNA was used in $1.2-2$ $\mathrm{GHz}$ measurement, which has $39 \mathrm{~dB}$ gain and $0.9 \mathrm{~dB}$ noise figure. The other was used in $2-18 \mathrm{GHz}$ measurement, which has $43 \mathrm{~dB}$ gain and $1.9 \mathrm{~dB}$ noise figure. The receiver system temperature was less than $300 \mathrm{~K}$ after including contribution from a 20-meter coaxial cable and the Agilent E4440A spectrum analyzer.

This RFI survey focused not only on "strong" interference and transmitters which potentially threaten the linearity of cryogenic receivers of the Shanghai 65 meter telescope, but also on "weak" RFI that may obscure weak astronomical sources of interest. The overall assessments can be exemplified by Figure 1, on which only the horizontal polarization measurement is plotted. Red and blue colored curves represent two different signal levels, the maximum and the mean value, respectively. Here, the original monitoring data have been converted into spectral flux density in $\mathrm{dB}\left(\mathrm{Wm}^{-2} \mathrm{~Hz}^{-1}\right)$. In general, new site evaluation in October 2009 is consistent with that made in May 2008, implying a relatively stable radio environment at the Sheshan site.

\section{Summary}

RFI has become a critical issue to the sites of radio telescopes in China. We understand that even if at the moment only little interference is found at some relatively low frequency range, it may not be true in the future as communications and other services move to higher frequencies. Therefore, some more work must be done in order to protect the RFI environment of the telescope sites.

More frequent RFI monitoring is necessary to ensure a reliable assessment at Shanghai Sheshan telescope site. On the one hand, we will take every measure to deal with any possible new interference within allocated spectral bands. This includes some regulatory issues at the administration level in terms of the spectrum management for radio astronomy. On the other hand, we will work closely with experts on the development of relevant algorithms and techniques for RFI mitigation on our 65 meter system, such as superconducting filters. We will also pay attention to the EMC (Electro magnetic Compatibility) testing of the actuator designed for active surface of the Shanghai 65 meter telescope.

\section{Acknowledgments}

This work was supported in part by the Knowledge Innovation Program of the Chinese Academy of Sciences (Grant No. KJCX1-YW-18) and the Scientific Program of Shanghai Municipality (08DZ1160100). Z.-Q Shen acknowledges the support by the National Natural Science Foundation of China (grants 10625314, 10633010 and 10821302). 